\title{
Unified Finite Series Approximation of FSO Performance over Strong Turbulence Combined with Various Pointing Error Conditions
}

\author{
Kug-Jin Jung, Student Member, IEEE, Sung Sik Nam, Member, IEEE, \\ Mohamed-Slim Alouini, Fellow Member, IEEE, and Young-Chai Ko, Senior Member, IEEE
}

\begin{abstract}
In this paper, we investigate both the bit error rate (BER) and outage performance of free-space optical (FSO) links over strong turbulence combined with various pointing error conditions. Considering atmospheric turbulence and pointing errors as main factors that deteriorate the quality of an optical link, we obtain a unified finite series approximation of the composite probability density function, which embraces generalized pointing error models. This approximations leads to new unified formulas for the BER and outage capacity of an FSO link, which account for the two possible detection mechanisms of intensity modulation/direct detection and heterodyne detection. Selected simulation results confirm that the newly derived approximations can give precise predictions of both the average BER and the outage capacity of FSO communication that are generally applicable to all environments.
\end{abstract}

\section{INTRODUCTION}

Spectrum scarcity is becoming the primary concern in radio frequency (RF) communication. Optical wireless communication (OWC) is a potential solution. This method uses the unlicensed optical spectrum to transmit signal. Specifically, Freespace optical (FSO) communication as long range outdoor OWC, establishes point-to-point communication links through the atmosphere. Unlike wireless RF communication, FSO communication does not require a license with high bandwidth and high capacity. Due to these attractive characteristics, FSO has attracted increasing interest over the last several decades.

Despite the advantages FSO communication has over RF communication, it still faces important challenges. One of the main concerns is atmospheric turbulence, which causes random fluctuations in the temporal and spatial irradiance of the optical beam, which may significantly degrade performance [2]. Several statistical models have been proposed to describe the conditions of weak and strong turbulence [2], [3]. Moreover, considering communication for mobile platforms such as drones, sea vessels, and automobiles, motion can lead to misalignment between the transmitter and receiver. Such misalignment causes various forms of pointing errors that do not arise in an FSO link between fixed devices [4].

This is an extended version of papers which were presented in ICC (2018) [1].

K.-J. Jung, S. S. Nam (co-first author) and Y. -C. Ko are with the School of Electrical Engineering, Korea University, Seoul 02841, South Korea (E-mail: $<$ kug0860, ssnam, koyc $>@$ korea.ac.kr). M.-S. Alouini is with the Computer, Electrical and Mathematical Science and Engineering Division (CEMSE), King Abdullah University of Science and Technology (KAUST), Thuwal, Saudi Arabia (E-mail: slim.alouini@kaust.edu.sa)
Pointing error can be analyzed in terms of the displacement of the laser beam along the horizontal and vertical directions which are assumed to follow independent, and identically distributed zero-mean Gaussian distributions [5]. Pointing errors consist of two components: boresight and jitter. The boresight is the fixed displacement between center of the beam and the center of the detector. The jitter is random offset of beam center at the detector plane. Previous studies have presented several models of pointing error that differ in their inclusion of boresight components and whether the jitter effects the two displacement directions identically or not [4], [6].

The performance analysis in the presence of the combined effects of atmospheric turbulence and pointing error using composite probability density function (PDF) has been studied in [6]-[26]. Especially, the analysis has been conducted for strong atmospheric turbulence in various situations of system model and detection techniques [6]-[23]. Authors in [6][12] derived bit error rate and capacity in case of single link FSO system. To mitigate the performance degradation by pointing errors and atmospheric turbulence, researcher groups have applied existing RF communication techniques to FSO systems [13]-[21]. In [13]-[17], the authors investigated error performance of FSO system with relay and authors in [18], [19] combined multiple-input multiple-output (MIMO) system with FSO to improve performance and derived the error rate. Also, hybrid FSO/RF system has been studied to improve reliability of FSO system and the error rate was derived in [20], [21]. However, the research groups in [6][21] assumed that the pointing error model follows Rayleigh distribution in case of strong turbulence and utilized composite PDF derived in [9]. These results represent only one specific pointing error and are expressed in terms of special function, such as Meijer's G-function; therefore, the channel models are not suited to describing the various types of errors that occur in mobile platform-based environments. Furthermore, most existing studies have focused on intensity modulation and direct detection (IM/DD) technique [7], [9], [10], [14], [17], [20]-[23]. Even though IM/DD with on-off keying (OOK) is usually utilized in optical wireless system, coherent communications have been also considered as alternative detection mode. Heterodyne detection (HD) and its performance across atmospheric turbulence has been investigated in [8], [11], [13], [26] with limited pointing error model (i.e. Rayleigh model).

Also, considering more general pointing error models combined with strong turbulence, researchers have derived average 
bit error rate (BER) results in [22], [23] and ergodic capacity in [24]. In [22], the authors assumed pointing error model of Rician distribution, obtained a composite PDF for strong turbulence as an approximate finite sum and conducted error rate analysis of on-off keying (OOK) signaling with intensity modulation and direct detection (IM/DD). Similarly in [23], the pointing error model of Hoyt distribution was considered and the authors investigated error performance of OOK with strong turbulence. Authors in [24] conducted unified capacity analysis over various types of turbulence with Rician pointing error model. However, these results are also limited to a particular model of the pointing error and IM/DD detection technique. Recently in [25], a threshold-based multiple optical signal selection scheme (TMOS) for free-space optical wavelength division multiplexing systems was proposed. Here, the average BER was derived over weak turbulence channel model (i.e., Log-normal channel model) taking into considerations the impact of fixed point-to-point pointing error but these derived results are limited to the proposed scheme, especially for weak turbulence conditions.

To the best of our best knowledge, no studies have modeled comprehensively the various types of pointing errors that may occur in a FSO link between mobile platforms, especially in the strong turbulence conditions. Accordingly, no uniform expressions that consider strong turbulence and various pointing error models not only for IM/DD but also for HD technique have been published. Although unified expression that treats the BER performance of both detection techniques were presented in [12], this work is limited to a pointing error model based on the Rayleigh distribution.

In this context, this paper considers both the BER and outage capacity over strong turbulence conditions with the various pointing error models presented in [4]. These models are especially intended for the analysis of mobile FSO links. More specifically, we statistically analyze composite PDFs in unified finite series forms. Using the unified composite PDFs, we investigate both the BER and outage capacity performance of an FSO system as the unified expressions based on both the IM/DD and HD technique. Based on our results, it is possible to analyze the performance of FSO link considering detection techniques, and modulation types with changing transceiver environment.

The remainder of this paper is organized as follows. Section II introduces the system model and reviews the available statistical models for strong atmospheric turbulence and pointing error. In Section III, we derive a composite channel gain PDF for each pointing error model with strong turbulence, and we present a unified expression for the composite PDF. Then, Section IV derives the unified composite PDF for the signal-to-noise ratio (SNR) for the IM/DD and HD schemes. Both the average BER and the outage capacity are derived from these results in Section V-A and Section V-B, respectively. Section VI presents numerical results that validate our analysis, and Section VII summarizes our findings.

\section{System And Channel Model}

In a typical FSO communication link, the transmitter modulates the instantaneous intensity of an optical beam to transmit data. In this work, the laser beams propagate through a strong turbulence channel with additive white Gaussian noise (AWGN) in the presence of pointing errors. The received optical signal is converted into an electrical signal at the photodetector. The signal is influenced by atmospheric attenuation, atmospheric turbulence, pointing error, and AWGN. We can express the received signal $y$ as

$$
y=\eta_{e} h x+n,
$$

where $x$ is the transmitted signal, $\eta_{e}$ is the effective photoelectric conversion ratio, $h$ is the channel gain, and $n$ is the signal-independent AWGN with variance $N_{0}$. We assume that atmospheric fading and the pointing error are independent, and that the channel gain $h$ can be modeled as $h=h_{l} h_{a} h_{p}$ where $h_{l}$ represents path-loss, $h_{a}$ is the atmospheric fading loss factor, and $h_{p}$ is the pointing error loss factor. Note that atmospheric fading loss factor $h_{a}$ and the pointing error loss factor $h_{p}$ are both random variables, whereas the path-loss $h_{l}$ is a constant given as $h_{l}=\exp (-\sigma z)$ where $\sigma$ is attenuation coefficient and $z$ is link distance [27].

\section{A. Atmospheric Turbulence}

Several statistical models have been proposed for atmospheric fading according to degree of the turbulence. In this paper, we assume that the signal is transmitted by a plane wave with strong turbulence, and that $h_{a}$ can be modeled as a Gamma-Gamma (ГГ) random variable (RV). Then the PDF of $h_{a}$ can be given as [3]

$$
f_{h_{a}}\left(h_{a}\right)=\frac{2(\alpha \beta)^{\frac{\alpha+\beta}{2}}}{\Gamma(\alpha) \Gamma(\beta)} h_{a}^{\frac{\alpha+\beta}{2}-1} K_{\alpha-\beta}\left(2 \sqrt{\alpha \beta h_{a}}\right),
$$

where $\Gamma(\cdot)$ is the Gamma function [28, eq.(8.310.1)], $K_{v}(\cdot)$ is the $v$ th-order modified Bessel function of the second kind [28, eq.(8.432.2)], and $\alpha$, and $\beta$ are the effective number of small-scale and large-scale eddies in the scattering environment, respectively. In [3], $\alpha$ and $\beta$ are given as $\alpha=\left[\exp \left(\frac{0.49 \sigma_{R}{ }^{2}}{\left(1+1.11 \sigma_{R}{ }^{12 / 5}\right)^{7 / 6}}\right)-1\right]^{-1}, \beta=$ $\left[\exp \left(\frac{0.51 \sigma_{R}{ }^{2}}{\left(1+0.69 \sigma_{R}{ }^{12 / 5}\right)^{5 / 6}}\right)-1\right]^{-1}$, where $\sigma_{R}^{2}$ is a metric for the strength of turbulence, referred to as the Rytov variance. It is defined as $\sigma_{R}{ }^{2}=1.23 k^{7 / 6} C_{n}{ }^{2} z^{11 / 6}$ where $k$ is the optical wavenumber, $z$ is the propagation distance, and $C_{n}{ }^{2}$ is the index of refraction structure parameter of atmosphere. In the case of strong turbulence, the Rytov variance satisfies the condition $\sigma_{R}^{2} \geq 0.3$. The $n$th moment of $h_{a}$ is given by [4]

$$
\mathbb{E}\left[h_{a}^{n}\right]=\frac{\Gamma(\alpha+n) \Gamma(\beta+n)}{(\alpha \beta)^{n} \Gamma(\alpha) \Gamma(\beta)} .
$$

\section{B. Pointing Error}

Assuming a Gaussian beam with beamwidth $w_{z}$ that propagates over a distance $z$ from transmitter to detector with 
TABLE I

PDF AND FIRST MOMENTS OF POINTING ERROR MODELS

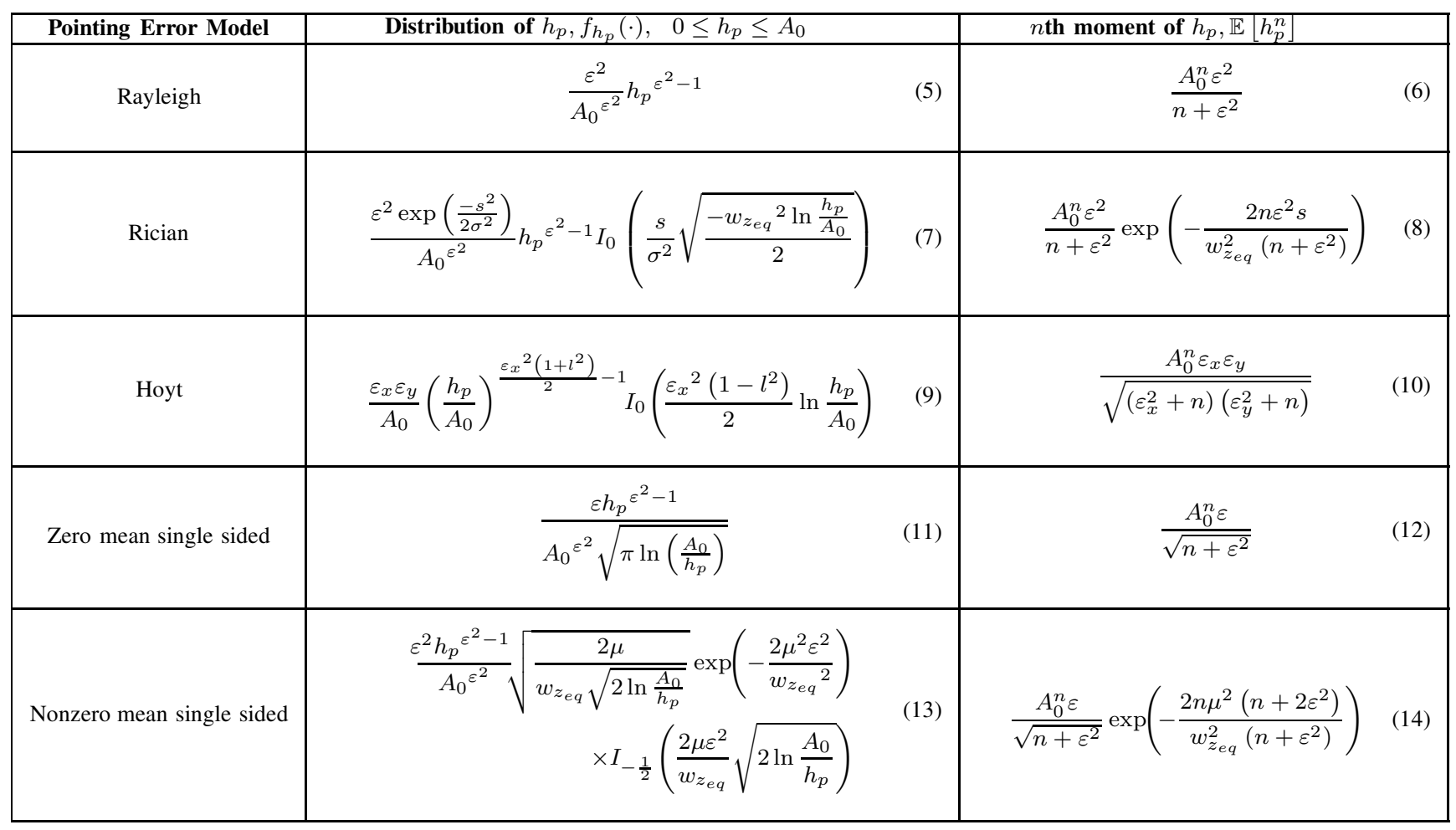
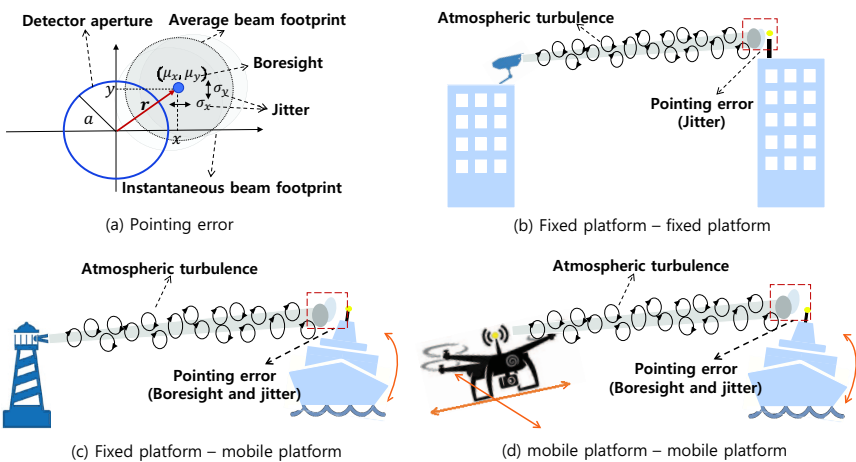

(d) mobile platform - mobile platform

Fig. 1. Types of pointing error in FSO links

aperture radius $a$, the fraction of the collected power at the receiver can be approximated by [6]

$$
h_{p}(r ; z) \approx A_{0} \exp \left(-\frac{2 r^{2}}{w_{z_{e q}}{ }^{2}}\right),
$$

where $r$ is the radial displacement between the centers of the beam and detector, $A_{0}$ is the fraction of collected power at $r=0$, and $w_{z_{e q}}$ is the equivalent beamwidth defined as $w_{z_{e q}}{ }^{2}=w_{z}{ }^{2} \frac{\sqrt{A_{0} \pi}}{2 v \exp \left(-v^{2}\right)}$. We have $A_{0}=[\operatorname{erf}(v)]^{2}$, where $v=\sqrt{\frac{a^{2} \pi}{2 w_{z}^{2}}}$ is the ratio between the aperture radius and beamwidth, and erf $(x)=\frac{2}{\sqrt{\pi}} \int_{0}^{x} e^{-t^{2}} d t$ is the error function. The approximation in (4) is valid when $w_{z}>6 a$ and can be attained by typical FSO communication systems. As Fig. 1(a) shows, the radial displacement vector can be expressed as

$\mathbf{r}=[x, y]^{T}$, where $x$ and $y$, respectively denote the horizontal and the vertical displacement of the beam in the detector plane. Assuming that the displacements follow independent Gaussian distributions along the axes, we can express $x$ and $y$ as $r_{x} \sim \mathcal{N}\left(\mu_{x}, \sigma_{x}^{2}\right)$, and $r_{y} \sim \mathcal{N}\left(\mu_{y}, \sigma_{y}^{2}\right)$, respectively. Then the radial displacement $r=|\mathbf{r}|=\sqrt{x^{2}+y^{2}}$ follows the Beckmann distribution [29]. Depending on the type of boresight and jitter values, pointing error can be modeled in five different ways. Pointing error models can be classified according to whether the boresight value is zero or not. In case of jitter, the error model can be categorized by the number of its value (i.e., single-sided jitter or double-sided jitter) and double-sided jitter model can also be divided by whether the two jitter values are identical or not. The Beckman distribution, which represents the general form of radial displacement, can be specialized to various distributions for each type of pointing error model, as shown below. Table I gives the PDF and the average of $h_{p}$ for each distribution [4]. Fig 1(b), Fig 1(c), and Fig 1(d) illustrate various FSO link environments according to the mobility of Tx and Rx. Typically, the pointing error between fixed platforms (Fig 1(b)) can be modeled as case 1) below. For mobile platforms (Fig 1 (c) and Fig 1 (d)), the pointing error can be modeled as one of the cases 2) through 5 ) below depending on the mobility of the mobile platform and the environmental conditions.

1) Zero Boresight and Identical Jitters: In this case, since both $x$ and $y$ have zero mean and identical variance (i.e., $\mu_{x}=$ $\mu_{y}=0$ and $\sigma_{x}=\sigma_{y}=\sigma$ ), the radial displacement $r$ follows a Rayleigh distribution. The PDF and average of $h_{p}$ are given as (5) and (6) in Table I where $\varepsilon=\frac{w_{z_{e q}}}{2 \sigma}$. 
2) Nonzero Boresight and Identical Jitters: When both displacements have nonzero mean with different values and common variance (i.e., $\mu_{x} \neq \mu_{y}$ and $\sigma_{x}=\sigma_{y}=\sigma$ ), $r$ is a Rician distributed RV. (7) and (8) in Table I show the PDF and the average of $h_{p}$, respectively, where $s=\sqrt{\mu_{x}^{2}+\mu_{y}^{2}}$ is the boresight displacement and $I_{\nu}(\cdot)$ denotes the $\nu$ th-order modified Bessel function of the first kind [28, eq.(8.431.1)].

3) Zero Boresight and Non-Identical Jitters: If $x$ and $y$ have zero mean and non-equal variances (i.e., $\mu_{x}=\mu_{y}=0$, $\sigma_{x} \neq \sigma_{y}$ and $\sigma_{x}<\sigma_{y}$ ), $r$ is a Hoyt distributed RV. The PDF and average of $h_{p}$ can be derived as (9) and (10) in Table I where $\varepsilon_{x}=\frac{w_{z_{e q}}}{2 \sigma_{x}}, \varepsilon_{y}=\frac{w_{z_{e q}}}{2 \sigma_{y}}$, and $l=\frac{\sigma_{x}}{\sigma_{y}}=\frac{\varepsilon_{y}}{\varepsilon_{x}} \quad(l<1)$.

4) Zero Boresight and Single Jitter: In this case, the displacement occurs in only the horizontal or the vertical axis and has zero mean (i.e., $\mu_{x}=\mu_{y}=0$, and $\sigma_{x}=\sigma, \sigma_{y}=0$ or $\sigma_{x}=0, \sigma_{y}=\sigma$ ). The radial displacement $r$ is a centralchi RV with one degree of freedom. The PDF of $h_{p}$ and its average are given by (11) and (12) in Table I.

5) Nonzero Boresight and Single Jitter: Last, if the displacement is only in one direction with nonzero mean (i.e., $\mu_{x}=\mu, \mu_{y}=0, \sigma_{x}=\sigma, \sigma_{y}=0$ or $\mu_{x}=0, \mu_{y}=\mu$, $\left.\sigma_{x}=0, \sigma_{y}=\sigma\right), r$ is a non-central-chi RV with one degree of freedom. In Table I, the PDF of $h_{p}$ is given by (13) and the average is written as (14).

\section{Composite PDF of Channel Gain}

\section{A. Special Cases}

The PDF of channel gain $h=h_{l} h_{a} h_{p}$ can be given as [6]

$$
\begin{aligned}
f_{h}(h) & =\int f_{h \mid h_{a}}\left(h \mid h_{a}\right) f_{h_{a}}\left(h_{a}\right) d h_{a} \\
& =\int \frac{1}{h_{a} h_{l}} f_{h_{p}}\left(\frac{h}{h_{a} h_{l}}\right) f_{h_{a}}\left(h_{a}\right) d h_{a} .
\end{aligned}
$$

Substituting (2) into (15), the composite PDF for GammaGamma turbulence channel can be written as

$$
\begin{aligned}
f_{h}(h)=\int & \frac{1}{h_{a} h_{l}} f_{h_{p}}\left(\frac{h}{h_{a} h_{l}}\right) \frac{2(\alpha \beta)^{\frac{\alpha+\beta}{2}}}{\Gamma(\alpha) \Gamma(\beta)} \\
& \times h_{a}^{\frac{\alpha+\beta}{2}-1} K_{\alpha-\beta}\left(2 \sqrt{\alpha \beta h_{a}}\right) d h_{a} .
\end{aligned}
$$

1) Nonzero Boresight and Identical Jitters: The authors in [22] obtained a finite series approximation for a composite PDF with this type of pointing error under the condition $\varepsilon^{2}>$ $\max (\alpha, \beta)$. The composite PDF $f_{\text {Rician }}(h)$ is derived with a series expansion of the modified Bessel function of the second kind [30, eq. (03.04.06.0002.01)]. This PDF is given as (17) where $J=\left\lfloor\varepsilon^{2}-\max (\alpha, \beta)\right\rfloor$ and $\lfloor x\rfloor$ denotes the largest integer that is less than $x$. This result can be specialized to derive PDF for a Rayleigh distribution when the boresight $s$ is set to zero $(s=0)$.

2) Zero Boresight and Non-Identical Jitters: Substituting (9) into (16), the composite PDF in the case of Hoyt distribu- tion can be written as

$$
\begin{aligned}
f_{\text {Hoyt }}(h)= & \int_{\frac{h}{A_{0} h_{l}}}^{\infty} \frac{1}{h_{a} h_{l}} \frac{\varepsilon_{x} \varepsilon_{y}}{A_{0}}\left(\frac{h}{A_{0} h_{a} h_{l}}\right)^{\frac{\varepsilon^{2}\left(1+l^{2}\right)}{2}-1} \\
\times & I_{0}\left(\frac{\varepsilon_{x}^{2}\left(1-l^{2}\right)}{2} \ln \frac{h}{A_{0} h_{a} h_{l}}\right) \frac{2(\alpha \beta)^{\frac{\alpha+\beta}{2}}}{\Gamma(\alpha) \Gamma(\beta)} \\
& \times h_{a}^{\frac{\alpha+\beta}{2}-1} K_{\alpha-\beta}\left(2 \sqrt{\alpha \beta h_{a}}\right) d h_{a} .
\end{aligned}
$$

If $x=\frac{1}{2} \ln \frac{A_{0} h_{a} h_{l}}{h}$, then we can write $h_{a}=\frac{h e^{2 x}}{A_{0} h_{l}}$. Applying change of variables from $h_{a}$ to $x$ and after some mathematical manipulation, we can express (18) as

$$
\begin{aligned}
& f_{\text {Hoyt }}(h)= \\
& \begin{aligned}
\frac{4 \varepsilon_{x} \varepsilon_{y}\left(\frac{\alpha \beta}{A_{0} h_{l}}\right)^{\frac{\alpha+\beta}{2}} h^{\frac{\alpha+\beta}{2}}-1}{\Gamma(\alpha) \Gamma(\beta)} \int_{0}^{\infty} \exp \left(x\left(\alpha+\beta-\varepsilon_{x}^{2}\left(1+l^{2}\right)\right)\right) \\
\quad \times I_{0}\left(\varepsilon_{x}^{2}\left(l^{2}-1\right) x\right) K_{\alpha-\beta}\left(2 \sqrt{\frac{\alpha \beta h}{A_{0} h_{l}}} e^{x}\right) d x .
\end{aligned}
\end{aligned}
$$

Using a series representation of the modified Bessel function of the second kind [30, eq. (03.04.06.0002.01)]

$$
\begin{aligned}
K_{v}(x) & =\frac{\pi}{2 \sin (\pi v)}\left[\sum_{k=0}^{\infty} \frac{1}{\Gamma(k-v+1) k !}\left(\frac{x}{2}\right)^{2 k-v}\right. \\
& \left.-\sum_{k=0}^{\infty} \frac{1}{\Gamma(k+v+1) k !}\left(\frac{x}{2}\right)^{2 k+v}\right], \quad[v \notin \mathbb{Z}, \quad|x|<\infty]
\end{aligned}
$$

where $v$ in (20) corresponds to $\alpha-\beta$ in (19) and swapping the integral and summation, (19) can be expressed as (21). Then, to solve the integral in (21), we use the integral identity [28, eq. (6.611.4)]

$$
\int_{0}^{\infty} e^{-a x} I_{v}(b x) d x=\frac{b^{-v}\left[a-\sqrt{a^{2}-b^{2}}\right]^{v}}{\sqrt{a^{2}-b^{2}}},
$$

$$
[\operatorname{Re} v>-1, \quad \operatorname{Re} a>|\operatorname{Re} b|]
$$

where $I_{v}(\cdot)$ is the modified Bessel function of the first kind of $\nu$-th order. Since the integral identity (22) requires that the summation index $j$ in (21) be less than or equal to $J=$ $\left\lfloor\varepsilon_{y}^{2}-\max (\alpha, \beta)\right\rfloor,(21)$ is developed into the approximate expression (23). Also, the approximation (23) is valid under the condition $\varepsilon_{y}^{2}>\max (\alpha, \beta)$.

3) Zero Boresight and Single Jitter: Substituting (11) into (16), we can write the composite PDF for the pointing error model based on the zero-mean single-sided Gaussian distribution as

$$
\begin{aligned}
& f_{\text {zero-single }}(h)=\int_{\frac{h}{A_{0} h_{l}}}^{\infty} \frac{1}{h_{a} h_{l}} \frac{\varepsilon\left(\frac{h}{h_{a}}\right)^{\varepsilon^{2}-1}}{A_{0} \varepsilon^{2} \sqrt{\pi \ln \left(\frac{A_{0} h_{a} h_{l}}{h}\right)}} \\
& \times \frac{2(\alpha \beta)^{\frac{\alpha+\beta}{2}}}{\Gamma(\alpha) \Gamma(\beta)} h_{a}{ }^{\frac{\alpha+\beta}{2}-1} K_{\alpha-\beta}\left(2 \sqrt{\alpha \beta h_{a}}\right) d h_{a} .
\end{aligned}
$$

Let $x=\sqrt{\ln \frac{A_{0} h_{a} h_{l}}{h}}$ and it can be also written as $h_{a}=\frac{h e^{x^{2}}}{A_{0} h_{l}}$. 


$$
\begin{aligned}
f_{\text {Rician }}(h) \approx \sum_{j=0}^{J}\left\{\frac { 1 } { j ! } ( \frac { \alpha \beta } { A _ { 0 } h _ { l } } ) ^ { j } \left(\frac{\pi \varepsilon^{2}\left(\frac{\alpha \beta}{A_{0} h_{l}}\right)^{\beta} \exp \left(-\frac{s^{2}}{2 \sigma_{s}{ }^{2}}-\frac{s^{2} \varepsilon^{2} / \sigma_{s}{ }^{2}}{2 \beta-2 \varepsilon^{2}+2 j}\right) h^{\beta-1+j}}{\sin ((\alpha-\beta) \pi) \Gamma(\alpha) \Gamma(\beta) \Gamma(j-(\alpha-\beta)+1)\left(\varepsilon^{2}-\beta-j\right)}\right.\right. \\
\left.\left.-\frac{\pi \varepsilon^{2}\left(\frac{\alpha \beta}{A_{0} h_{l}}\right)^{\alpha} \exp \left(-\frac{s^{2}}{2 \sigma_{s}{ }^{2}}-\frac{s^{2} \varepsilon^{2} / \sigma_{s}{ }^{2}}{2 \alpha-2 \varepsilon^{2}+2 j}\right) h^{\alpha-1+j}}{\sin ((\alpha-\beta) \pi) \Gamma(\alpha) \Gamma(\beta) \Gamma(j+(\alpha-\beta)+1)\left(\varepsilon^{2}-\alpha-j\right)}\right)\right\} .
\end{aligned}
$$

$$
\begin{aligned}
& f_{\text {Hoyt }}(h)=\frac{2 \pi \varepsilon_{x} \varepsilon_{y}\left(\frac{\alpha \beta}{A_{0} h_{l}}\right)^{\frac{\alpha+\beta}{2}} h^{\frac{\alpha+\beta}{2}-1}}{\Gamma(\alpha) \Gamma(\beta) \sin (\pi(\alpha-\beta))} \\
& \times \sum_{j=0}^{\infty}\left(\frac{1}{\Gamma(j-(\alpha-\beta)+1) j !}\left(\frac{\alpha \beta h}{A_{0} h_{l}}\right)^{j-\frac{\alpha-\beta}{2}} \int_{0}^{\infty} \exp \left(x\left(2 \beta+2 j-\varepsilon_{x}^{2}\left(1+l^{2}\right)\right)\right) I_{0}\left(\varepsilon_{x}^{2}\left(l^{2}-1\right) x\right) d x\right. \\
& \left.-\frac{1}{\Gamma(j+(\alpha-\beta)+1) j !}\left(\frac{\alpha \beta h}{A_{0} h_{l}}\right)^{j+\frac{\alpha-\beta}{2}} \int_{0}^{\infty} \exp \left(x\left(2 \alpha+2 j-\varepsilon_{x}^{2}\left(1+l^{2}\right)\right)\right) I_{0}\left(\varepsilon_{x}^{2}\left(l^{2}-1\right) x\right) d x\right) .
\end{aligned}
$$

$$
\begin{array}{r}
f_{\text {Hoyt }}(h) \approx \sum_{j=0}^{J}\left\{\frac { 1 } { j ! } ( \frac { \alpha \beta } { A _ { 0 } h _ { l } } ) ^ { j } \left(\frac{\pi \varepsilon_{x} \varepsilon_{y}\left(\frac{\alpha \beta}{A_{0} h_{l}}\right)^{\beta} h^{\beta-1+j}}{\sin ((\alpha-\beta) \pi) \Gamma(\alpha) \Gamma(\beta) \Gamma(j-(\alpha-\beta)+1) \sqrt{\left(\varepsilon_{x}^{2}-\beta-j\right)\left(\varepsilon_{y}^{2}-\beta-j\right)}}\right.\right. \\
\left.\left.-\frac{\pi \varepsilon_{x} \varepsilon_{y}\left(\frac{\alpha \beta}{A_{0} h_{l}}\right)^{\alpha} h^{\alpha-1+j}}{\sin ((\alpha-\beta) \pi) \Gamma(\alpha) \Gamma(\beta) \Gamma(j+(\alpha-\beta)+1) \sqrt{\left(\varepsilon_{x}^{2}-\alpha-j\right)\left(\varepsilon_{y}^{2}-\alpha-j\right)}}\right)\right\} .
\end{array}
$$

We change the variables of (24) from $h_{a}$ to $x$ and simplify the expression to

$$
\begin{aligned}
& f_{\text {zero-single }}(h)= \\
& \begin{aligned}
\frac{4 \varepsilon\left(\frac{\alpha \beta}{A_{0} h_{l}}\right)^{\frac{\alpha+\beta}{2}} h^{\frac{\alpha+\beta}{2}}-1}{\sqrt{\pi} \Gamma(\alpha) \Gamma(\beta)} \int_{0}^{\infty} \exp \left(x^{2}\left(\frac{\alpha+\beta}{2}-\varepsilon^{2}\right)\right) \\
\quad \times K_{\alpha-\beta}\left(2 \sqrt{\frac{\alpha \beta h}{A_{0} h_{l}} \exp \left(x^{2}\right)}\right) d x .
\end{aligned}
\end{aligned}
$$

Using the series representation that was applied in (20), the modified Bessel function of the second kind in (25) can be replaced with an infinite series. By swapping the integral and summation, (25) is transformed into (26). To derive a closedform expression from the integral in (26), we use an integral identity [28, eq. (3.321.3)]

$$
\int_{0}^{\infty} e^{-q^{2} x^{2}} d x=\frac{\sqrt{\pi}}{2 q}, \quad[q>0]
$$

where the summation index $j$ in (26) must be less than or equal to $J=\left\lfloor\varepsilon^{2}-\max (\alpha, \beta)\right\rfloor$. Accordingly, the infinite summation in (26) is transformed into the finite series approximation as (28) and the approximation is also valid under the condition $\varepsilon^{2}>\max (\alpha, \beta)$.

4) Nonzero Boresight and Single Jitter: In this case, the composite PDF can be obtained by substituting (13) into (16) as

$$
\begin{aligned}
& f_{\text {nonzero-single }}(h)= \\
& \int_{\frac{h}{A_{0} h_{l}}}^{\infty} \frac{1}{h_{a} h_{l}} \frac{\varepsilon^{2}}{A_{0}^{\varepsilon^{2}}}\left(\frac{h}{h_{a} h_{l}}\right)^{\varepsilon^{2}-1} \sqrt{\frac{2 \mu}{w_{z_{e q}} \sqrt{2 \ln \frac{A_{0} h_{a} h_{l}}{h}}}} \\
& \quad \times \exp \left(-\frac{2 \mu^{2} \varepsilon^{2}}{w_{z_{e q}}^{2}}\right) I_{-\frac{1}{2}}\left(\frac{2 \mu \varepsilon^{2}}{w_{z_{e q}}} \sqrt{2 \ln \frac{A_{0} h_{a} h_{l}}{h}}\right) \\
& \quad \times \frac{2(\alpha \beta)^{\frac{\alpha+\beta}{2}}}{\Gamma(\alpha) \Gamma(\beta)} h_{a}{ }^{\frac{\alpha+\beta}{2}-1} K_{\alpha-\beta}\left(2 \sqrt{\alpha \beta h_{a}}\right) d h_{a} .
\end{aligned}
$$

If $x=\sqrt{2 \ln \frac{A_{0} h_{a} h_{l}}{h}}$, then we can set $h_{a}=\frac{h e^{\frac{x^{2}}{2}}}{A_{0} h_{l}}$. By using a change of variables and algebraic manipulation, (29) reduces to

$$
\begin{aligned}
& f_{\text {nonzero-single }}(h)=\frac{2 \varepsilon^{2}\left(\frac{\alpha \beta}{A_{0} h_{l}}\right)^{\frac{\alpha+\beta}{2}} h^{\frac{\alpha+\beta}{2}-1}}{\Gamma(\alpha) \Gamma(\beta) \exp \left(\frac{2 \mu^{2} \varepsilon^{2}}{w_{z_{e q}}}\right)} \\
& \times \int_{0}^{\infty} \sqrt{x} \exp \left(\frac{x^{2}}{2}\left(\frac{\alpha+\beta}{2}-\varepsilon^{2}\right)\right) \\
& \times I_{-\frac{1}{2}}\left(\frac{2 \mu \varepsilon^{2} x}{w_{z_{e q}}}\right) K_{\alpha-\beta}\left(2 \sqrt{\frac{\alpha \beta h}{A_{0} h_{l}} \exp \left(\frac{x^{2}}{2}\right)}\right) d x .
\end{aligned}
$$

Then, we apply the series expansion (20) to (30) to transform the modified Bessel function into series form as (31) where $P(j, \alpha)=\int_{0}^{\infty} \sqrt{x} \exp \left(\frac{x^{2}}{2}\left(\alpha+j-\varepsilon^{2}\right)\right) I_{-\frac{1}{2}}\left(\frac{2 \mu \varepsilon^{2} x}{w_{z_{e q}}}\right) d x$. Applying a change of variable rule $y=x^{2}$, we have $P(j, \alpha)=\frac{1}{2} \int_{0}^{\infty} y^{-\frac{1}{4}} \exp \left(-\frac{y\left(\varepsilon^{2}-\alpha-j\right)}{2}\right) I_{-\frac{1}{2}}\left(\frac{2 \mu \varepsilon^{2} \sqrt{y}}{w_{z_{e q}}}\right) d y$. 


$$
\begin{array}{r}
f_{\text {zero-single }}(h)=\frac{2 \pi \varepsilon\left(\frac{\alpha \beta}{A_{0} h_{l}}\right)^{\frac{\alpha+\beta}{2}} h^{\frac{\alpha+\beta}{2}-1}}{\sqrt{\pi} \Gamma(\alpha) \Gamma(\beta) \sin (\pi(\alpha-\beta))} \sum_{j=0}^{\infty}\left(\frac{1}{\Gamma(j-(\alpha-\beta)+1) j !}\left(\frac{\alpha \beta h}{A_{0} h_{l}}\right)^{j-\frac{\alpha-\beta}{2}} \int_{0}^{\infty} \exp \left(x^{2}\left(\beta+j-\varepsilon^{2}\right)\right) d x\right. \\
\left.-\frac{1}{\Gamma(j+(\alpha-\beta)+1) j !}\left(\frac{\alpha \beta h}{A_{0}}\right)^{j+\frac{\alpha-\beta}{2}} \int_{0}^{\infty} \exp \left(x^{2}\left(\alpha+j-\varepsilon^{2}\right)\right) d x\right) .
\end{array}
$$

$$
\begin{array}{r}
f_{\text {zero-single }}(h) \approx \sum_{j=0}^{J}\left\{\frac { 1 } { j ! } ( \frac { \alpha \beta } { A _ { 0 } h _ { l } } ) ^ { j } \left(\frac{\pi \varepsilon\left(\frac{\alpha \beta}{A_{0} h_{l}}\right)^{\beta} h^{\beta-1+j}}{\sin ((\alpha-\beta) \pi) \Gamma(\alpha) \Gamma(\beta) \Gamma(j-(\alpha-\beta)+1) \sqrt{\varepsilon^{2}-\beta-j}}\right.\right. \\
\left.\left.-\frac{\pi \varepsilon\left(\frac{\alpha \beta}{A_{0} h_{l}}\right)^{\alpha} h^{\alpha-1+j}}{\sin ((\alpha-\beta) \pi) \Gamma(\alpha) \Gamma(\beta) \Gamma(j+(\alpha-\beta)+1) \sqrt{\varepsilon^{2}-\alpha-j}}\right)\right\} .
\end{array}
$$

In the following derivation, by using an integral identity [28, eq. (6.643.2)]

$$
\begin{aligned}
& \int_{0}^{\infty} x^{u-\frac{1}{2}} e^{-a x} I_{2 v}(2 b \sqrt{x}) d x= \\
& \frac{\Gamma\left(u+v+\frac{1}{2}\right)}{\Gamma(2 v+1)} b^{-1} \exp \left(\frac{b^{2}}{2 a}\right) a^{-u} M_{-u, v}\left(\frac{b^{2}}{a}\right) \\
& {\left[\operatorname{Re}\left(u+v+\frac{1}{2}\right)>0\right]}
\end{aligned}
$$

where $M_{u, v}(\cdot)$ is the Whittaker function [28, eq.(9.220.4)], the integral form $P(j, \alpha)$ can be expressed as

$$
\begin{array}{r}
P(j, \alpha)=\frac{w_{z_{e q}}}{2 \mu \varepsilon^{2}} \exp \left(\frac{\mu^{2} \varepsilon^{4}}{\left(\varepsilon^{2}-\alpha-j\right) w_{z_{e q}}{ }^{2}}\right)\left(\frac{\varepsilon^{2}-\alpha-j}{2}\right)^{-\frac{1}{4}} \\
\times M_{-\frac{1}{4},-\frac{1}{4}}\left(\frac{2 \mu^{2} \varepsilon^{4}}{\left(\varepsilon^{2}-\alpha-j\right) w_{z_{e q}}{ }^{2}}\right)
\end{array}
$$

The integral condition in (32) requires that the summation index $j$ in (31) be less than or equal to $J=\left\lfloor\varepsilon^{2}-\max (\alpha, \beta)\right\rfloor$. We use another identity [30, eq. (03.04.06.0002.01)]

$$
M_{-m-\frac{1}{2}, m}(z)=\exp \left(\frac{z}{2}\right) z^{\frac{2 m+1}{2}}
$$

to express (33) as

$$
P(j, \alpha)=\frac{1}{2} \exp \left(\frac{2 \mu^{2} \varepsilon^{4}}{\left(\varepsilon^{2}-\alpha-j\right) w_{z_{e q}}{ }^{2}}\right) \sqrt{\frac{2 w_{z_{e q}}}{\left(\varepsilon^{2}-\alpha-j\right) \mu \varepsilon^{2}}} .
$$

Finally, substituting (35) into (31), we obtain a composite PDF for the non-zero mean single-sided Gaussian distribution as (36). Since the summation index $j$ is truncated by the integral condition in (32), the result in (36) is an approximation that is valid under the condition $\varepsilon^{2}>\max (\alpha, \beta)$.
TABLE II

PARAMETERS FOR UNIFIED EXPRESSION

\begin{tabular}{|c|c|c|c|c|c|c|}
\hline Pointing Error Model & $\varepsilon_{x}$ & $\varepsilon_{y}$ & $s$ & $d$ & $\rho_{x}$ & $\rho_{y}$ \\
\hline Rayleigh & $\varepsilon$ & $\varepsilon$ & 0 & 2 & 1 & 1 \\
\hline Rician & $\varepsilon$ & $\varepsilon$ & $s$ & 2 & 1 & 1 \\
\hline Hoyt & $\varepsilon_{x}$ & $\varepsilon_{y}$ & 0 & 2 & 1 & 1 \\
\hline Zero mean single sided & $\varepsilon$ & 0 & 0 & 1 & 1 & 0 \\
\hline Nonzero mean single sided & $\varepsilon$ & 0 & $\mu$ & 1 & 1 & 0 \\
\hline
\end{tabular}

\section{B. Unified Expression}

Using the similarity of terms, (17), (23), (28), and (36) can be unified as

$$
\begin{array}{r}
f(h) \approx \sum_{j=0}^{J}\left\{\frac { 1 } { j ! } ( \frac { \alpha \beta } { A _ { 0 } h _ { l } } ) ^ { j } \left(v_{j}\left(\alpha, \beta \mid \varepsilon_{x}, \varepsilon_{y}, s, d, \rho_{x}, \rho_{y}\right) h^{\beta-1+j}\right.\right. \\
\left.\left.+v_{j}\left(\beta, \alpha \mid \varepsilon_{x}, \varepsilon_{y}, s, d, \rho_{x}, \rho_{y}\right) h^{\alpha-1+j}\right)\right\},
\end{array}
$$

where $v_{j}\left(\alpha, \beta \mid \varepsilon_{x}, \varepsilon_{y}, s, d, \rho_{x}, \rho_{y}\right)$ can generally be expressed as (38) and can be specialized for each of the pointing error models listed in Table II.

\section{Composite PDF OF SNR}

\section{A. $I M / D D$}

For IM/DD detection, the SNR is defined as

$$
\gamma=\frac{\eta_{e}^{2} h^{2}}{N_{0}}
$$

where $\eta_{e}$ denotes the effective photoelectric conversion ratio. Then, the average electrical SNR can be written as

$$
\mu_{I M / D D}=\frac{\eta_{e}^{2} \mathbb{E}_{h}\left[h^{2}\right]}{N_{0}},
$$

where $\mathbb{E}[\cdot]$ denotes the expectation operator. Since $h_{a}$ and $h_{p}$ are statistically independent processes, and $h_{l}$ is deterministic, (40) becomes

$$
\mu_{I M / D D}=\frac{\eta_{e}^{2} h_{l}^{2} \mathbb{E}_{h_{p}}\left[h_{p}^{2}\right] \mathbb{E}_{h_{a}}\left[h_{a}^{2}\right]}{N_{0}}
$$




$$
\begin{aligned}
& f_{\text {nonzero-single }}(h)=\frac{\pi \varepsilon^{2}\left(\frac{\alpha \beta}{A_{0} h_{l}}\right)^{\frac{\alpha+\beta}{2}} \exp \left(-\frac{2 \mu^{2} \varepsilon^{2}}{w_{z_{e q}}{ }^{2}}\right) h^{\frac{\alpha+\beta}{2}-1}}{\Gamma(\alpha) \Gamma(\beta) \sin (\pi(\alpha-\beta))} \\
& \times \sum_{j=0}^{\infty}\left(\frac{1}{\Gamma(j-(\alpha-\beta)+1) j !}\left(\frac{\alpha \beta h}{A_{0} h_{l}}\right)^{j-\frac{\alpha-\beta}{2}} P(j, \beta)-\frac{1}{\Gamma(j+(\alpha-\beta)+1) j !}\left(\frac{\alpha \beta h}{A_{0} h_{l}}\right)^{j+\frac{\alpha-\beta}{2}} P(j, \alpha)\right) .
\end{aligned}
$$

$$
\begin{aligned}
f_{\text {nonzero-single }}(h) \approx & \sum_{j=0}^{J}\left\{\frac { 1 } { j ! } ( \frac { \alpha \beta } { A _ { 0 } h _ { l } } ) ^ { j } \left(\frac{\pi \varepsilon\left(\frac{\alpha \beta}{A_{0} h_{l}}\right)^{\beta} \exp \left(-\frac{\mu^{2}}{2 \sigma_{s}{ }^{2}}-\frac{\mu^{2} \varepsilon^{2} / \sigma_{s}{ }^{2}}{2 \beta-2 \varepsilon^{2}+2 j}\right) h^{\beta-1+j}}{\sin ((\alpha-\beta) \pi) \Gamma(\alpha) \Gamma(\beta) \Gamma(j-(\alpha-\beta)+1) \sqrt{\varepsilon^{2}-\beta-j}}\right.\right. \\
& \left.\left.-\frac{\pi \varepsilon\left(\frac{\alpha \beta}{A_{0} h_{l}}\right)^{\alpha} \exp \left(-\frac{\mu^{2}}{2 \sigma_{s}{ }^{2}}-\frac{\mu^{2} \varepsilon^{2} / \sigma_{s}{ }^{2}}{2 \beta-2 \varepsilon^{2}+2 j}\right) h^{\alpha-1+j}}{\sin ((\alpha-\beta) \pi) \Gamma(\alpha) \Gamma(\beta) \Gamma(j+(\alpha-\beta)+1) \sqrt{\varepsilon^{2}-\alpha-j}}\right)\right\} .
\end{aligned}
$$

$$
\begin{aligned}
& v_{j}\left(\alpha, \beta \mid \varepsilon_{x}, \varepsilon_{y}, s, d, \rho_{x}, \rho_{y}\right) \\
& =\frac{\pi\left(\varepsilon_{x}+1-\rho_{x}\right)^{\rho_{x}}\left(\varepsilon_{y}+1-\rho_{y}\right)^{\rho_{y}}\left(\frac{\alpha \beta}{A_{0} h_{l}}\right)^{\beta} \exp \left(-\frac{s^{2}}{2 \sigma_{s}{ }^{2}}-\frac{s^{2}\left(\varepsilon_{x}+1-\rho_{x}\right)^{\frac{2 \rho_{x}}{d}}\left(\varepsilon_{y}+1-\rho_{y}\right) \frac{2 \rho_{y}}{d} / \sigma_{s}{ }^{2}}{2 \beta-2\left(\varepsilon_{x}+1-\rho_{x}\right)^{\frac{2 \rho_{x}}{d}}\left(\varepsilon_{y}+1-\rho_{y}\right)^{\frac{\rho \rho y y}{d}}+2 j}\right)}{\sin ((\alpha-\beta) \pi) \Gamma(\alpha) \Gamma(\beta) \Gamma(j-(\alpha-\beta)+1)\left(\varepsilon_{x}{ }^{2}-\beta-j\right)^{\frac{\rho_{x}}{2}}\left(\varepsilon_{y}{ }^{2}-\beta-j\right)^{\frac{\rho_{y}}{2}}} .
\end{aligned}
$$

Substituting (3) into (41), and utilizing $\Gamma(z+n)=z^{n} \Gamma(z)$, we have

$$
\mu_{I M / D D}=\frac{\eta_{e}^{2} h_{l}^{2} \mathbb{E}_{h_{p}}\left[h_{p}^{2}\right]}{N_{0}} .
$$

Substituting (6), (8), (10), (12), and (14) into (42) for each pointing error model case, and assuming $\varepsilon^{2} \gg 2$, we obtain

$$
\mu_{I M / D D}=\frac{\eta_{e}^{2} A_{0}^{2} h_{l}^{2} c^{2}}{N_{0}},
$$

where $c=1$ if the pointing error model has zero boresight, such as the Rayleigh, Hoyt, and zero mean singlesided Gaussian distribution. If the pointing error model has nonzero boresight such as Rician and nonzero mean singlesided Gaussian distribution, $c$ can be given as, respectively

$$
\begin{aligned}
c_{\text {Rician }} & =\exp \left(-\frac{2 s^{2}}{w_{z_{e q}}{ }^{2}}\right), \\
c_{\text {nonzero-single }} & =\exp \left(-\frac{2 \mu^{2}}{w_{z_{e q}}{ }^{2}}\right) .
\end{aligned}
$$

Combining (39) with (43), the channel gain $h$ can be written in respect to average electrical SNR, $\mu_{I M / D D}$ as

$$
h=A_{0} h_{l} c \sqrt{\frac{\gamma}{\mu_{I M / D D}}} .
$$

Applying RV transformation of (45) into (37), the SNR PDF for the IM/DD detection scheme can be derived as (46) in Table III.

\section{B. Heterodyne}

For HD scheme, the SNR is given as

$$
\gamma=\frac{\eta_{e} h}{N_{0}}
$$

Similarly, we can express the average electrical SNR as

$$
\mu_{H D}=\frac{\eta_{e} A_{0} h_{l} c}{N_{0}}
$$

where $c$ follows the same rule as in the case of IM/DD detection, and the channel gain $h$ can be expressed as

$$
h=\frac{A_{0} h_{l} c \gamma}{\mu_{H D}} .
$$

Utilizing RV transformation of (49), the resulting SNR PDF for the HD technique is given as (50) in Table III.

Both the PDFs in (46) and (50) can be combined, yielding the unified expression (51) in Table III, where $r$ is a parameter that indicates the type of detection technique (i.e., $r=2$ represents IM/DD and $r=1 \mathrm{HD})$.

\section{Performance Analysis}

\section{A. Error Rate Performance}

BER for a given SNR in the case of IM/DD or HD can be written as [31]

$$
\operatorname{BER}(\gamma)=\frac{\Gamma(p, q \gamma)}{2 \Gamma(p)},
$$

where $\Gamma(\cdot, \cdot)$ represents incomplete gamma function and $p$ and $q$ are the parameters that define the type of detection mechanism and modulation type, respectively, as

$$
\begin{gathered}
p= \begin{cases}1, & \text { for IM/DD, } \\
\frac{1}{2,} & \text { for HD, }\end{cases} \\
q= \begin{cases}1, & \text { for PSK } \\
\frac{1}{2,} & \text { for FSK. }\end{cases}
\end{gathered}
$$


TABLE III

COMPOSITE PDF OF SNR

\begin{tabular}{|c|c|c|}
\hline Detection Technique & PDF & \\
\hline IM/DD & $\begin{aligned} f_{\gamma_{I M / D D}}(\gamma)= & \frac{1}{2} \sum_{j=0}^{J}\left\{\frac{1}{j !}\left(\frac{\alpha \beta c}{\sqrt{\mu_{I M / D D}}}\right)^{j}\left(v_{j}\left(\alpha, \beta \mid \varepsilon_{x}, \varepsilon_{y}, s, d, \rho_{x}, \rho_{y}\right)\left(\frac{A_{0} h_{l} c}{\sqrt{\mu_{I M / D D}}}\right)^{\beta} \gamma^{\frac{\beta+j}{2}-1}\right.\right. \\
& \left.\left.+v_{j}\left(\beta, \alpha \mid \varepsilon_{x}, \varepsilon_{y}, s, d, \rho_{x}, \rho_{y}\right)\left(\frac{A_{0} h_{l} c}{\sqrt{\mu_{I M / D D}}}\right)^{\alpha} \gamma^{\frac{\alpha+j}{2}}-1\right)\right\}\end{aligned}$ & (46) \\
\hline Heterodyne & $\begin{array}{r}f_{\gamma_{H} D}(\gamma)=\sum_{j=0}^{J}\left\{\frac{1}{j !}\left(\frac{\alpha \beta c}{\mu_{H D}}\right)^{j}\left(v_{j}\left(\alpha, \beta \mid \varepsilon_{x}, \varepsilon_{y}, s, d, \rho_{x}, \rho_{y}\right)\left(\frac{A_{0} h_{l} c}{\mu_{H D}}\right)^{\beta} \gamma^{\beta+j-1}\right.\right. \\
\left.\left.+v_{j}\left(\beta, \alpha \mid \varepsilon_{x}, \varepsilon_{y}, s, d, \rho_{x}, \rho_{y}\right)\left(\frac{A_{0} h_{l} c}{\mu_{H D}}\right)^{\alpha} \gamma^{\alpha+j-1}\right)\right\}\end{array}$ & (50) \\
\hline $\begin{array}{c}\text { Unified } \\
(r=2 \text { for IM/DD, } \\
r=1 \text { for HD) }\end{array}$ & $\begin{aligned} f_{\gamma}(\gamma)=\frac{1}{r} \sum_{j=0}^{J}\left\{\frac{1}{j !}\left(\frac{\alpha \beta c}{\mu_{r} \frac{1}{r}}\right)^{j}(\right. & v_{j}\left(\alpha, \beta \mid \varepsilon_{x}, \varepsilon_{y}, s, d, \rho_{x}, \rho_{y}\right)\left(\frac{A_{0} h_{l} c}{\mu_{r} \frac{1}{r}}\right)^{\beta} \gamma^{\frac{\beta+j}{r}}-1 \\
& \left.\left.+v_{j}\left(\beta, \alpha \mid \varepsilon_{x}, \varepsilon_{y}, s, d, \rho_{x}, \rho_{y}\right)\left(\frac{A_{0} h_{l} c}{\mu_{r} \frac{1}{r}}\right)^{\alpha} \gamma^{\frac{\alpha+j}{r}}-1\right)\right\}\end{aligned}$ & (51) \\
\hline
\end{tabular}

Hence, the average BER can be obtained by integrating (52) with respect to the PDF of $\gamma$ as

$$
P_{e}=\int_{0}^{\infty} \frac{\Gamma(p, q \gamma)}{2 \Gamma(p)} f_{\gamma}(\gamma) d \gamma
$$

After substituting (51) into (53), we swap the integral and the summation with some mathematical manipulation to transform (53) into (54). The integral form in (54) can be found in [28, eq. (6.455.1)]

$$
\begin{aligned}
\int_{0}^{\infty} & x^{u-1} \Gamma(v, a x) e^{-b x} d x= \\
& \frac{a^{v} \Gamma(u+v)}{u(a+b)^{u+v}}{ }_{2} F_{1}\left(1, u+v ; u+1 ; \frac{b}{a+b}\right)
\end{aligned}
$$

where ${ }_{2} F_{1}$ denotes the Gaussian hypergeometric function. Thus, after calculating the integral using (55), the average BER can finally be written as (56).

\section{B. Outage Capacity}

Since the coherence time of FSO channels is 1-100 msec, it is considerably longer than the typical bit interval, which is on the order of $1 \mathrm{nsec}$. Consequently, the FSO channel can be modeled as a slow-fading channel, in which the channel state is fixed over a large number of transmitted bits [32]. We assume perfect channel state information at the receiver and that data of $R_{0}$ bits/channel is used at the transmitter. Given specific SNR $\gamma$, the achievable channel capacity is given by

$$
\mathcal{C}(\gamma)=\log _{2}(1+\lambda \gamma)
$$

where $\lambda$ indicates the type of detection mechanism as [33]

$$
\lambda= \begin{cases}\frac{e}{2 \pi}, & \text { for IM/DD, } \\ 1, & \text { for HD. }\end{cases}
$$

In the case of IM/DD, $\mathcal{C}$ represents a tight lower bound on channel capacity, and represents the exact capacity in case of HD.

For such slow-fading FSO channels, outages occur if $R_{0}$ exceeds $\mathcal{C}$. The outage probability is defined as

$$
P_{\text {out }}\left(R_{0}\right)=\operatorname{Pr}\left(\mathcal{C}(\gamma)<R_{0}\right) \text {. }
$$

Substituting (57) into (58), we find

$$
P_{\text {out }}\left(R_{0}\right)=\operatorname{Pr}\left(\log _{2}(1+\lambda \gamma)<R_{0}\right),
$$

and since $\log _{2}(\cdot)$ increases monotonically with respect to $\gamma$,

$$
P_{\text {out }}\left(R_{0}\right)=\operatorname{Pr}\left(\gamma<\frac{2^{R_{0}}-1}{\lambda}\right) \text {. }
$$

At last, the outage probability can be calculated as

$$
P_{\text {out }}\left(R_{0}\right)=\int_{0}^{\frac{2^{R_{0}-1}}{\lambda}} f_{\gamma}(\gamma) d \gamma \text {. }
$$

Hence, we can utilize the unified composite PDF for SNR (51) to derive the outage probability for different pointing error models and detection techniques. By substituting (51) into (61), we formulate the outage probability as (62).

\section{NUMERICAL RESUlTS}

In this section, we carry out both the error rate performance and the outage capacity of an FSO communication system and validate our analytical results by comparing with exact MonteCarlo simulations. All the simulations adopt the system setting shown in Table IV, Table V, and Table VI. Table IV shows the typical parameter settings used in many practical terrestrial FSO communication systems [22]. The parameters to describe the atmospheric turbulence condition (i.e., strong turbulence and moderate turbulence) and each pointing error model are listed in Table V and Table VI respectively. To investigate 


$$
\begin{array}{r}
P_{e}=\frac{1}{2 r \Gamma(p)} \sum_{j=0}^{J}\left\{\frac { 1 } { j ! } ( \frac { \alpha \beta c } { \mu _ { r } ^ { \frac { 1 } { r } } } ) ^ { j } \left(v_{j}\left(\alpha, \beta \mid \varepsilon_{x}, \varepsilon_{y}, s, d, \rho_{x}, \rho_{y}\right)\left(\frac{A_{0} h_{l} c}{\mu_{r}^{\frac{1}{r}}}\right)^{\beta} \int_{0}^{\infty} \Gamma(p, q \gamma) \gamma^{\frac{\beta+j}{r}-1} d \gamma\right.\right. \\
+v_{j}\left(\beta, \alpha \mid \varepsilon_{x}, \varepsilon_{y}, s, d, \rho_{x}, \rho_{y}\right)\left(\frac{A_{0} h_{l} c}{\mu_{r}^{\frac{1}{r}}}\right)^{\alpha} \int_{0}^{\infty} \Gamma(p, q \gamma) \gamma^{\frac{a+j}{r}-1} d \gamma
\end{array}
$$

$$
\begin{aligned}
& P_{e}=\frac{1}{2 r \Gamma(p)} \sum_{j=0}^{J}\left\{\frac { 1 } { j ! } ( \frac { \alpha \beta c } { \mu _ { r } ^ { \frac { 1 } { r } } } ) ^ { j } \left(v_{j}\left(\alpha, \beta \mid \varepsilon_{x}, \varepsilon_{y}, s, d, \rho_{x}, \rho_{y}\right)\left(\frac{A_{0} h_{l} c}{\mu_{r}^{\frac{1}{r}}}\right)^{\beta} \frac{q^{p} \Gamma\left(\frac{\beta+j}{r}+p\right)}{\left(\frac{\beta+j}{r}\right) q^{\left(\frac{\beta+j}{r}+p\right)}} 2 F_{1}\left(1, \frac{\beta+j}{r}+p ; \frac{\beta+j}{r}+1 ; 0\right)\right.\right. \\
& \left.\left.+v_{j}\left(\beta, \alpha \mid \varepsilon_{x}, \varepsilon_{y}, s, d, \rho_{x}, \rho_{y}\right)\left(\frac{A_{0} h_{l} c}{\mu_{r}{ }^{\frac{1}{r}}}\right)^{\alpha} \frac{q^{p} \Gamma\left(\frac{\alpha+j}{r}+p\right)}{\left(\frac{\alpha+j}{r}\right) q^{\left(\frac{\alpha+j}{r}+p\right)}}{ }_{2} F_{1}\left(1, \frac{\alpha+j}{r}+p ; \frac{\alpha+j}{r}+1 ; 0\right)\right)\right\} .
\end{aligned}
$$

$$
\begin{array}{r}
P_{\text {out }}\left(R_{0}\right)=\frac{1}{r} \sum_{j=0}^{J}\left\{\frac { 1 } { j ! } ( \frac { \alpha \beta c } { \mu _ { r } ^ { \frac { 1 } { r } } } ) ^ { j } \left(v_{j}\left(\alpha, \beta \mid \varepsilon_{x}, \varepsilon_{y}, s, d, \rho_{x}, \rho_{y}\right)\left(\frac{A_{0} h_{l} c}{\mu_{r}^{\frac{1}{r}}}\right)^{\beta} \frac{r}{\beta+j}\left(\frac{2^{R_{0}}-1}{\lambda}\right)^{\frac{\beta+j}{r}}\right.\right. \\
\left.\left.\quad+v_{j}\left(\beta, \alpha \mid \varepsilon_{x}, \varepsilon_{y}, s, d, \rho_{x}, \rho_{y}\right)\left(\frac{A_{0} h_{l} c}{\mu_{r}^{\frac{1}{r}}}\right)^{\alpha} \frac{r}{\alpha+j}\left(\frac{2^{R_{0}}-1}{\lambda}\right)^{\frac{\alpha+j}{r}}\right)\right\}
\end{array}
$$

TABLE IV

PARAMETER SETTINGS

\begin{tabular}{|l|c|c|}
\hline Parameter & Symbol & Value \\
\hline Receiver radius & $a$ & $10 \mathrm{~cm}$ \\
Beamwidth & $w_{z}$ & $100 \mathrm{~cm}$ \\
Modulation type & $q$ & 1 \\
Attenuation coefficient & $\sigma$ & 1.10622 \\
Distance between Tx and Rx & $z$ & $0.1 \mathrm{~km}$ \\
\hline
\end{tabular}

TABLE V

TURBULENCE SETTINGS

\begin{tabular}{|c|c|c|}
\hline Turbulence strength & $\sigma_{R}^{2}$ & Parameter \\
\hline Strong & 6.0 & $\alpha=5.049, \beta=1.157$ \\
Moderate & 2.0 & $\alpha=3.993, \beta=1.702$ \\
\hline
\end{tabular}

the effect of boresight, we compare Rician, Zero mean, and Nonzero mean pointing error models with the same jitter value. In addition, to investigate how jitter degrades the signal, we compare the Rayleigh, Hoyt, Zero mean pointing error models with zero boresight.

In Fig. 2 to Fig. 5, the average BER curves are plotted against the average electrical SNR $\mu$ for different pointing error models and detection techniques. Fig. 2 and Fig. 3 give results for the IM/DD detection technique while Fig. 4 and Fig. 5 give results for the HD technique. We confirm that the unified average BER in (56) provides a precise evaluation within the scope of $\mu$ shown below. From Fig. 2, we find that the pointing error model that has the largest boresight value (Rician) shows the worst error rate performance and conversely, the Zero mean pointing error model that has zero boresight value performs the best in both turbulence conditions. In Fig. 3, we note that the different jitter values result in a slight gap between the pointing error models even though the jitters take maximum values within the scope allowed by summation index $j$. The pointing error model with the largest

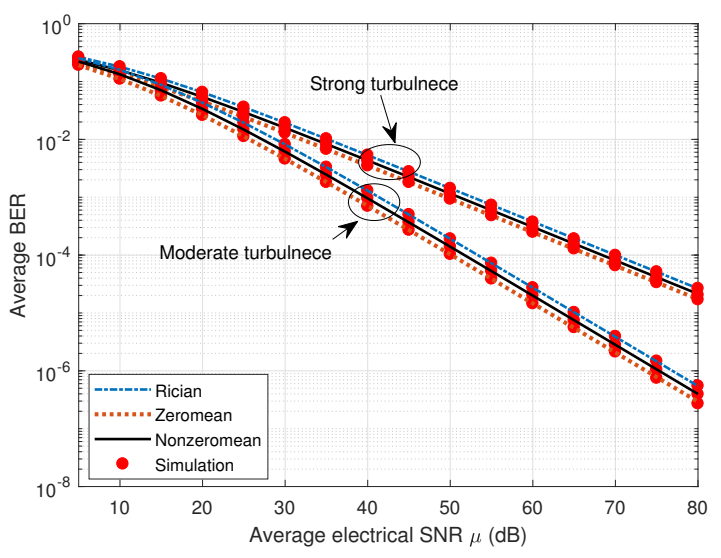

Fig. 2. Average BER of IM/DD with both moderate and strong turbulence for Rician, Zeromean, and Nonzeromean pointing error models

jitter value (Rayleigh) shows the worst performance among the pointing error models with zero boresight, regardless of the turbulence condition. Comparing Fig. 2 and Fig. 3, we also find that boresight affects performance degradation more than jitter. We can infer that the misalignment between Tx and $\mathrm{Rx}$ has a greater effect on the energy collected at the receiver aperture than the random offset of the beam center.

In Fig. 6, average BER curves are plotted against the distance between $\mathrm{Tx}$ and $\mathrm{Rx}$ for both detection techniques. First, we can see that the characteristics of the boresight and jitter values explained above still apply. In addition, the performance for both detection techniques degrades as the distance increases; however, the HD technique yields higher performance when compared to IM/DD, within the scope of the distance shown below.

In Fig. 7 and Fig. 8, the outage probability is plotted for 
TABLE VI

POINTING ERROR SETTINGS

\begin{tabular}{|c|c|c|c|c|c|c|}
\hline \multicolumn{2}{|c|}{ Axis } & \multicolumn{3}{c|}{ Double-sided } & \multicolumn{2}{c|}{ Single-sided } \\
\hline Error type & Rayleigh & Rician & Hoyt & Zero mean & Nonzero mean \\
\hline \multirow{2}{*}{ Boresight $(\mathrm{cm})$} & $\mu_{x}$ & 0 & 30 & 0 & 0 & 30 \\
\cline { 2 - 6 } & $\mu_{y}$ & 0 & 30 & 0 & 0 & 0 \\
\multirow{2}{*}{ Jitter $(\mathrm{cm})$} & $\sigma_{x}$ & 12 & 5 & 5 & 5 & 5 \\
\cline { 2 - 3 } & $\sigma_{y}$ & 12 & 5 & 12 & 0 & 0 \\
\hline
\end{tabular}

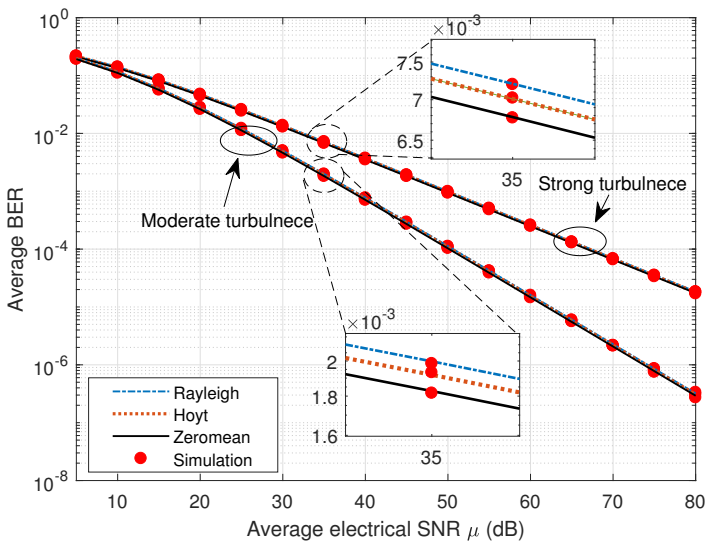

Fig. 3. Average BER of IM/DD with both moderate and strong turbulence for Rayleigh, Hoyt, and Zeromean pointing error models

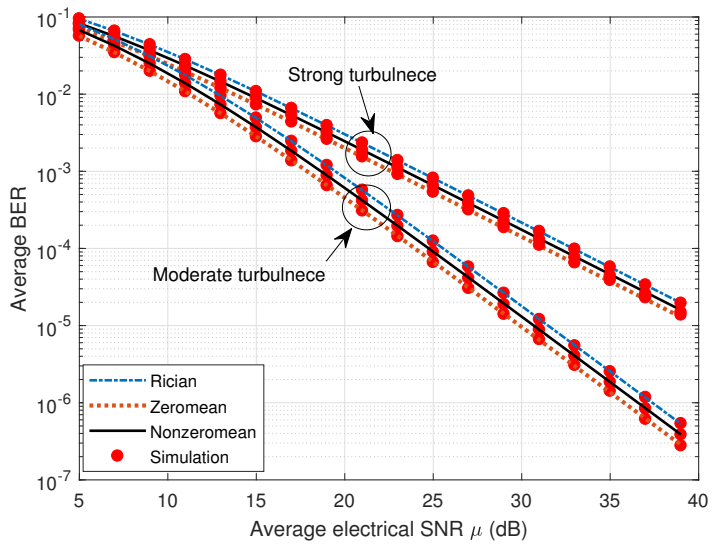

Fig. 4. Average BER of HD with both moderate and strong turbulence for Rician, Zeromean, and Nonzeromean pointing error models

IM/DD and HD techniques, respectively. As shown above, since the performance of HD is better than that of IM/DD, we can see that with same value of the capacity threshold $R_{0}$, the outage probability for IM/DD is much better than that for HD. In addition, the characteristics of pointing error models discussed above still apply for the case of outage probability.

\section{CONCLUSION}

In this paper, we studied both the BER and outage performance of a single FSO link over strong turbulence channel combined with various pointing error channel models which can occur in mobile platform-based environments. More specifically, by considering atmospheric turbulence and pointing errors as the main factors that deteriorate link performance,

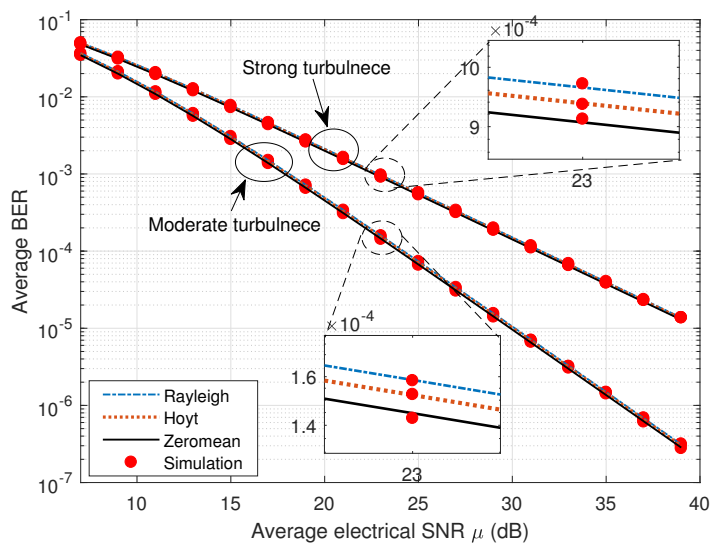

Fig. 5. Average BER of HD with both moderate and strong turbulence for Rayleigh, Hoyt, and Zeromean pointing error models

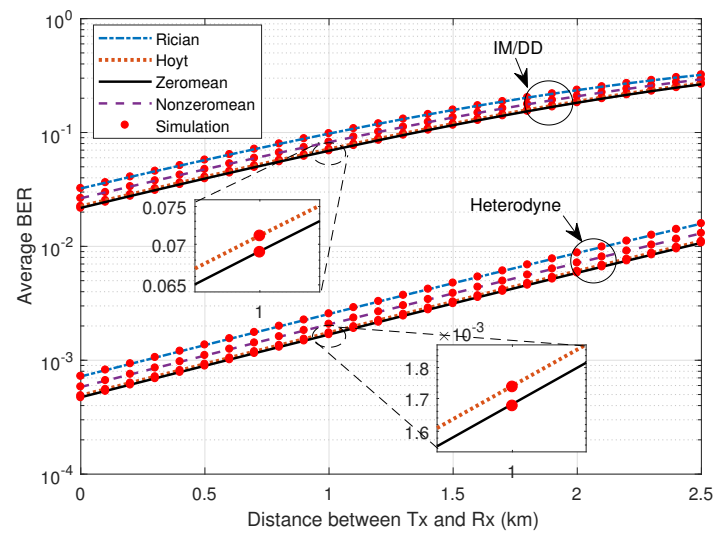

Fig. 6. Average BER of IM/DD in terms of the distance between Tx and Rx with strong turbulence model $(\alpha=5.049, \beta=1.157, \mu=25 \mathrm{~dB})$

we obtained unified PDFs that embrace generalized pointing error models. With these unified results, we derived the closedform results for the average BER and the outage capacity of an FSO link in the form of unified expressions based on both detection mechanisms (i.e., IM/DD and HD). Then, we used Monte-Carlo simulations to verify our analytical results under various FSO channel turbulence conditions and pointing errors and the derived simulation results agreed well with our analytical results.

\section{ACKNOWLEDGMENT}

This research was supported by the MSIT (Ministry of Science and ICT), Korea, under the ITRC (Information Technology Research Center) support program (IITP-2020-2015- 


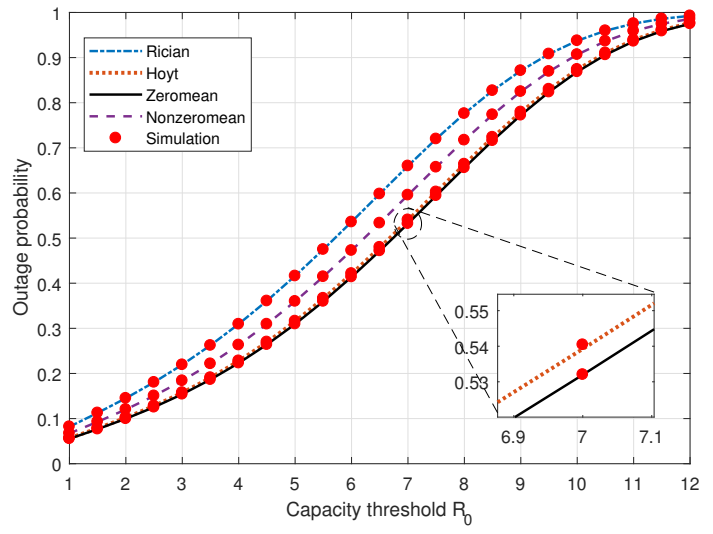

Fig. 7. Outage probability of IM/DD with strong turbulence $(\alpha=5.049, \beta=$ $1.157, \mu=25 \mathrm{~dB})$

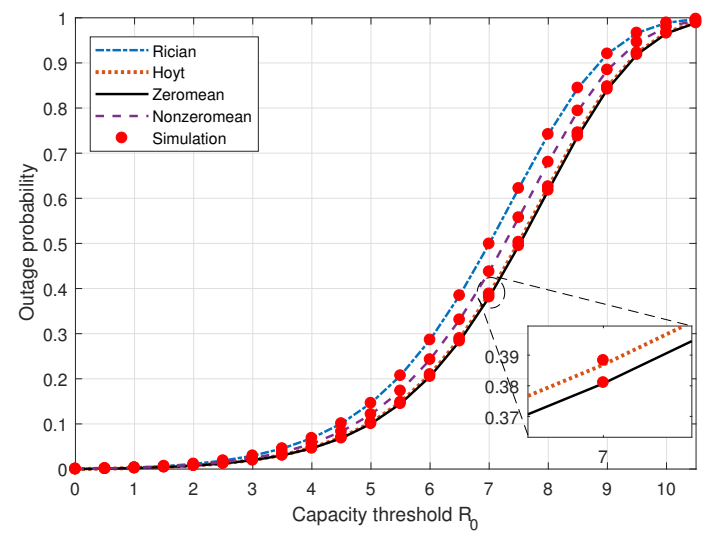

Fig. 8. Outage probability of HD with strong turbulence $(\alpha=5.049, \beta=$ $1.157, \mu=25 \mathrm{~dB})$

0-00385) supervised by the IITP (Institute for Information \& communications Technology Promotion)

\section{REFERENCES}

[1] K.-J. Jung, S. S. Nam, Y.-C. Ko, and M.-S. Alouini, "BER Performance of FSO Links Over Unified Channel Model for Pointing Error Models," in Proc. IEEE Int. Conf. Commun. (ICC) Workshops, Kanasas City, USA, May. 21-24, 2018.

[2] L. C. Andrews, R. L. Phillips, and C. Y. Hopen, Laser Beam Scintillation with Applications. SPIE press, 2011, vol. 99.

[3] M. Al-Habash, L. C. Andrews, and R. L. Phillips, "Mathematical model for the irradiance probability density function of a laser beam propagating through turbulent media," Opt Eng, vol. 62, no. 2, pp. 1554 1562, Feb. 2001.

[4] H. Al-Quwaiee, H. C. Yang and M. S. Alouini, "On the asymptotic ergodic capacity of FSO links with generalized pointing error model," in Proc. IEEE Int. Conf. Commun. (ICC), London, U.K., Jun. 2015, pp. $5072-5077$.

[5] S. Arnon, "Effects of atmospheric turbulence and building sway on optical wireless-communication systems," Opt. Lett., vol. 28, no. 12, pp. 129-131, Jan. 2003.

[6] A. Farid, and S. Hranilovic, "Outage capacity optimization for free-space optical links with pointing errors," IEEE/OSA J. Lightwave Technol., vol. 25, no. 7, pp. 1702-1710, July. 2007.

[7] L. L. Cao, M. Sheng, M. Feng and X. X. Xie, "BER performance of freespace optical communication in strong turbulence with pointing errors," Int. Conf. on Wire. Comm. and Sig. Proc. (WCSP), Huangshan, 2012, pp. 1-5.
[8] H. Sandalidis, Tsiftsis and G. Karagiannidis, "Optical wireless communications with heterodyne detection over turbulence channels with pointing errors," IEEE/OSA J. Lightwave Technol., vol. 27, no. 20, pp. 4440-4445, Oct. 2009.

[9] H. Sandalidis, T. Tsiftsis, G. Karagiannidis, and M. Uysal, "BER performance of FSO links over strong atmospheric turbulence channels with pointing errors," IEEE Commun. Lett., vol. 12, no. 1, pp. 44-46, Jan. 2008.

[10] G. Djordjevic, M. Petkovic, M. Spasic, and D. Antic, "Outage capacity of FSO link with pointing errors and link blockage," Opt. Express 24, pp. 219-230, Jan. 2016.

[11] T. Tsiftsis, "Performance of heterodyne wireless optical communication systems over gamma-gamma atmospheric turbulence channels," Electron. Lett., vol. 44, Feb. 2008.

[12] I. Ansari, F. Yilmaz, and M.-S. Alouini, "Performance analysis of FSO links over unified Gamma-Gamma turbulence channels," in Proc. IEEE VTC Spring, Glasgow, U.K., May 2015, pp. 1-5.

[13] E. Zedini and M. Alouini, "On the Performance of Multihop Heterodyne FSO Systems With Pointing Errors," in IEEE Photonics Journal, vol. 7 , no. 2, pp. 1-10, April 2015.

[14] R. Boluda-Ruiz, A. Garcia-Zambrana, C. Castillo-Vazquez, B. CastilloVazquez and S. Hranilovic, "Amplify-and-forward strategy using MRC reception over FSO channels with pointing errors," in IEEE J. Opt. Commun. Netw., vol. 10, no. 5, pp. 545-552, May 2018.

[15] P. Puri, P. Garg and M. Aggarwal, "Outage and Error Rate Analysis of Network-Coded Coherent TWR-FSO Systems," in IEEE Photonics Technology Letters, vol. 26, no. 18, pp. 1797-1800, 15 Sept.15, 2014.

[16] E. Zedini, H. Soury and M. Alouini, "Dual-Hop FSO Transmission Systems Over Gamma Gamma Turbulence With Pointing Errors," in IEEE Trans. Wirel. Commun., vol. 16, no. 2, pp. 784-796, Feb. 2017.

[17] E. Zedini and M. Alouini, "Multihop Relaying Over IM/DD FSO Systems With Pointing Errors," IEEE/OSA J. Lightwave Technol., vol. 33, no. 23, pp. 5007-5015, 1 Dec.1, 2015.

[18] M. R. Bhatnagar and Z. Ghassemlooy, "Performance Analysis of Gamma Gamma Fading FSO MIMO Links With Pointing Errors," IEEE/OSA J. Lightwave Technol., vol. 34, no. 9, pp. 2158-2169, 1 May1, 2016.

[19] M. R. Bhatnagar and S. Anees, "On the Performance of Alamouti Scheme in Gamma-Gamma Fading FSO Links With Pointing Errors," IEEE Wireless Communications Letters, vol. 4, no. 1, pp. 94-97, Feb. 2015.

[20] S. Sharma, A. S. Madhukumar and R. Swaminathan, "Effect of Pointing Errors on the Performance of Hybrid FSO/RF Networks," IEEE Access, vol. 7, pp. 131418-131434, 2019.

[21] A. Touati, A. Abdaoui, F. Touati, M. Uysal and A. Bouallegue, "On the effects of combined atmospheric fading and misalignment on the hybrid FSO/RF transmission," IEEE J. Opt. Commun. Netw., vol. 8, no. 10, pp. 715-725, Oct. 2016.

[22] F. Yang, J. Cheng, and T. Tsiftsis, "Free-space optical communication with nonzero boresight pointing errors," IEEE Trans. Commun., vol. 62, no. 2, pp. 713-725, Feb. 2014.

[23] W. Gappmair, S. Hranilovic, and E. Leitgeb, "OOK performance for terrestrial FSO links in turbulent atmosphere with pointing errors modeled by Hoyt distributions," IEEE Commun. Lett., vol. 15, no. 8, pp. 875-877, 2011.

[24] I. S. Ansari, M. Alouini and J. Cheng, "Ergodic Capacity Analysis of Free-Space Optical Links With Nonzero Boresight Pointing Errors," IEEE Trans. Wirel. Commun., vol. 14, no. 8, pp. 4248-4264, Aug. 2015.

[25] S. S. Nam, Y.-C. Ko, and M.-S. Alouini, "Threshold-based Multiple Optical Signal Selection Scheme for Free-Space Optical Wavelength Division Multiplexing Systems," IEEE J. Opt. Commun. Netw., Vol. 9, No. 12, pp. 1085-1096, Dec. 2017

[26] K. Kiasaleh, "Performance of coherent DPSK free-space optical communication systems in K-distributed turbulence," IEEE Trans. Commun., vol. 54, no. 4, pp. 604-607, Apr. 2006.

[27] M. Naboulsi, H. Sizun, and F. Fornel, "Propagation of optical and infrared waves in the atmosphere," in Proc. of the Union Radio Scientifique Internationale, 2005

[28] I. S. Gradshteyn and I. M. Ryzhik, Table of Integrals, Series, and Products, 6th ed. New York: Academic, 2000.

[29] P. Beckmann and A. Spizzichino, The Scattering of Electromagnetic Waves from Rough Surfaces. Artech House, 1987.

[30] Wolfram, "The wolfram functions site: http://functions.wolfram.com/," 2001.

[31] A. H. Wojnar, "Unknown bounds on performance in Nakagami channels," IEEE Trans. Commun., vol. 34, no. 1, pp. 22-24, Jan. 1986. 
[32] S. Hranilovic and F. R. Kschischang, "Capacity bounds for power- and band-limited optical intensity channels corrupted by Gaussian noise," IEEE Trans. Inform Theory, vol. 50, no. 5, pp. 784-795, May 2004.

[33] A. Chaaban, J. Morvan, and M.-S Alouini, "Free-Space Optical Communications: Capacity Bounds, Approximations, and a New Sphere-Packing Perspective," IEEE Trans. Commun., vol. 64, no. 3, pp. 1176-1191, March 2016 